\title{
HADAMARD DETERMINANTS, MÖBIUS FUNCTIONS, AND THE CHROMATIC NUMBER OF A GRAPH
}

\author{
BY HERBERT S. WILF ${ }^{1}$ \\ Communicated by Gian-Carlo Rota, April 5, 1968
}

The three subjects of the title are bound together by an interesting combinatorial identity, stated below as Theorem 1. This identity is itself rooted in special cases which have already appeared in the literature [3], but it could not have been stated in its full generality before the well known work of Rota [4] on Möbius functions. Indeed, we have here a good example of the unifying power of the concept of a Möbius function on a lattice in combinatorial analysis, for we are able to deduce as corollaries of this one piece of machinery (a) the evaluation of a determinant appearing in [1] as an example of a matrix of \pm 1 's whose determinant is "nearly" the Hadamard upper bound, (b) a theorem of [2] which is a partial generalization of (a), but is tied closely to a particular lattice, (c) perhaps most interesting, based on [4] we are able to exhibit a symmetric matrix depending on a parameter $\lambda$ which is singular if and only if a given graph cannot be colored in $\lambda$ colors. This matrix is reminiscent of another one found earlier by G. D. Birkhoff [5].

In reference [1] there appears an evaluation of the following determinant of \pm 1 's. Let $\alpha(i, j)$ denote the number of 1 's in common positions in the binary expansions of the integers $i$ and $j$, and define an $(n+1) \times(n+1)$ matrix $M(n)$ by

$$
M(n)_{i j}=(-1)^{\alpha(i, j)} \quad(i, j=0,1, \cdots, n) .
$$

The authors showed, by an inductive argument, that

$$
|\operatorname{det} M(n)|=2^{A(n)}
$$

where $A(n)=\alpha(0)+\alpha(1)+\cdots+\alpha(n)$ and $\alpha(m)$ is the sum of the binary digits of $m$. For large $n$, one has

$$
2^{A(n)} \geqq n^{n / 2(1-c / \log n)} \quad(c<1)
$$

so that (2) is reasonably close to the Hadamard upper bound $n^{n / 2}$.

In reference [2] there appears a generalization of the above results to lattices of subsets of a finite set, as well as investigations relating to bases other than 2 .

\footnotetext{
1 Research supported in part by the National Science Foundation.
} 
The germ of a more far-reaching generalization of these theorems is contained in a pair of problems in Pólya-Szegö ( $[3, \S \mathrm{VIII}$, Problems 32-33]), and the recent paper of Rota [4] on the use of the Möbius function of a lattice in combinatorial investigations.

Let there be given a partial order "@” for the integers $0,1, \cdots, n$. Define the "zeta-function" of the partial order as the $(n+1) \times(n+1)$ matrix

$$
\zeta_{i j}= \begin{cases}1 & j \preceq i \\ 0 & \text { otherwise. }\end{cases}
$$

Consider the matrix

$$
\Gamma=\zeta A \zeta^{T}
$$

where $A$ is a diagonal matrix with $a_{0}, a_{1}, \cdots, a_{n}$ on the diagonal, the $a_{i}$ being arbitrary. One evidently has

$$
\begin{aligned}
\operatorname{det} \Gamma & =(\operatorname{det} A)(\operatorname{det} \zeta)^{2} \\
& =a_{0} a_{1} \cdots a_{n} .
\end{aligned}
$$

On the other hand,

$$
\begin{aligned}
\Gamma_{i j} & =\sum_{k} \zeta_{i k} a_{k} \zeta_{j k} \\
& =\sum_{k \geqq i ; j\lfloor j j} a_{k} \quad(i, j=0,1, \cdots, n) .
\end{aligned}
$$

Let us define

$$
A_{i}=\sum_{k \leqq i} a_{k}
$$

and let us write $i \wedge j$ for the greatest lower bound ("meet") of $i$ and $j$. Then we have found that

$$
\left.\operatorname{det} A_{i \wedge j}\right]_{i, j=0}^{n}=a_{0} a_{1} a_{2} \cdots a_{n}
$$

where the $A_{i}$ and $a_{i}$ are related by (6). We can now eliminate the $a_{i}$ completely by inverting the formula (6). This gives

$$
a_{i}=\sum_{k \leqq i} \mu(i, k) A_{k} \quad(i=0,1, \cdots, n)
$$

where $\mu$ is the Möbius function of the lattice (see [4]), and is defined as the inverse of the matrix $\zeta$. Substituting (8) in (7) we find 
Theorem 1. Let $A_{0}, A_{1}, \cdots, A_{n}$ be arbitrary numbers, and let $\mu$ be the Möbius function of a lattice-ordering $\leqq$ of $\{0,1,2, \cdots, n\}$. Then

$$
\left.\operatorname{det} A_{i \wedge j}\right]_{i, j=0}^{n}=\prod_{m=0}^{n}\left\{\sum_{k \leqq m} \mu(m, k) A_{k}\right\} .
$$

Corollary 1. Let $A_{0}, \cdots, A_{n}$ be a sequence of \pm 1 , and let $\mu(m, k)$ be the Möbius function of a lattice partial ordering $\leqq$. Then the geometric mean of the numbers

$$
\left|\sum_{k \leqq m} \mu(m, k) A_{k}\right| \quad(m=0,1, \cdots, n)
$$

is at most $(n+1)^{1 / 2}$.

Proof. After (9), this is just Hadamard's inequality.

CoRollary 2. Equation (2) holds, where $M(n)$ is given by (1).

Proof. Define the ordering $\leqq$ by $a \leqq b$ if each binary digit of $a$ is $\leqq$ the corresponding digit of $\bar{b}$. The lattice $\{0,1, \ldots, n\}$ is then isomorphic to all subsets of a finite set, whose Möbius function is well known to be

$$
\mu(S, T)=(-1)^{\# S-\# T} \quad(T \subseteq S) .
$$

Hence

$$
\mu(a, b)=(-1)^{\alpha(b)-\alpha(a)} \quad(b \leqq a)
$$

where $\alpha(m)$ is the number of nonzero digits of $m$. Taking $A_{m}=(-1)^{\alpha(m)}$, we obtain from (9)

$$
\begin{aligned}
\left.\operatorname{det} A_{i \wedge j}\right]_{0}^{n} & \left.=\operatorname{det}(-1)^{\alpha(i \wedge j)}\right]_{0}^{n} \\
& \left.=\operatorname{det}(-1)^{\alpha(i, j)}\right]_{0}^{n} \\
& =\prod_{m=0}^{n}\left\{\sum_{k \leqq m}(-1)^{\alpha(m)-\alpha(k)}(-1)^{\alpha(k)}\right\} \\
& =(-2)^{A(n)}
\end{aligned}
$$

as required.

LEMMA 1. If $r \leqq s$ means that the b-ary digits of $r$ are $\leqq$ those of $s$, where $b>1$ is fixed, then 
$\mu(s, r)=(-1)^{\nu}$ if $\nu$ digits of $r$ are 1 less than those of $s$, the rest equal $=0$ otherwise.

Proof. We use Rota's crosscut theorem [4]. Fix $r, s, r \leqq s$. In the interval $r \leqq x \leqq s$ the crosscut of dual atoms consists of those numbers $x$ which agree with $s$ in all digits except one, where, in the latter, the digit of $x$ is 1 less than the corresponding digit of $s$. The meet of any collection of dual atoms is a number whose digits are at most 1 less than the digits of $s$. Hence if some digit of $s$ exceeds the corresponding digit of $r$ by 2 or more, $\mu(s, r)=0$, since no spanning subsets exist.

In the contrary case, exactly $\nu$ digits of $r$ are 1 less than the corresponding digit of $s$, the remaining digits being identical $(\nu \geqq 1)$. Then a spanning subset is a collection of dual atoms whose decreased digits are identical with these $\nu$. The number of spanning subsets of $k$ elements is thus

$$
\begin{array}{rlrl}
q_{k} & =1 & k=\nu, \\
& =0 & k \neq \nu .
\end{array}
$$

The crosscut theorem gives in this case

$$
\mu(s, r)=q_{2}-q_{3}+q_{4}-\cdots=(-1)^{\nu} .
$$

Now let $\alpha_{b}(m)$ denote the sum of the $b$-ary digits of $m$. Then, in the case where $\mu(s, r) \neq 0$ in Lemma $1, \mu(s, r)$ is equal to $(-1)^{\alpha_{b}(s)-\alpha_{b}(r)}$. Hence, put $A_{j}=(-1)^{\alpha_{b}(j)}$. Then from the theorem we find easily

Corollary 3. We have

$$
\left.\mid \operatorname{det}(-1)^{\alpha_{b}(i \wedge j)}\right]_{i, j=0}^{n} \mid=2^{\psi_{b}(n)}
$$

where $\psi_{b}(n)=\eta_{b}(0)+\cdots+\eta_{b}(n)$ and $\eta_{b}(m)$ is the number of nonzero $b$-ary digits of $m$.

As a final application, to graph coloring, we observe that Theorem 1 is in no way tied to the use of integers as members of the ordered set. Indeed, if $f$ is a function from an arbitrary lattice $L$ to, say, the complex numbers, we have

$$
\operatorname{det} f(S \wedge T)]_{S, T \in L}=\prod_{S \in L}\left\{\sum_{T \underline{\underline{S}}} \mu(S, T) f(T)\right\}
$$

as well as the dual form

$$
\operatorname{det} f(S \vee T)]_{S, T \in L}=\prod_{S \in L}\left\{\sum_{T \geqq S} \mu(T, S) f(T)\right\}
$$


which arises from using $\zeta^{T} a \zeta$ in (5). For the lattice $L$ take the collection of all bond-closed (see [4]) sets of edges of a given undirected finite graph $G$, and take

$$
f(T)=\lambda^{(n-r(T))} \quad(T \in L)
$$

where $n$ is the number of vertices of $G, r(T)$ is the rank of $T$ (i.e., number of vertices of $T$ minus the number of connected components) in the lattice $L$, and $\lambda$ is an indeterminate. Then, from [4] we know that

$$
p(\lambda, S)=\sum_{S \leqq T} \mu(T, S) f(T) \quad(S \in L)
$$

is the number of colorings of $G$ whose bond is $S$. Then from (11)

$$
\operatorname{det}\left[\lambda^{n-r(S \vee T)}\right]_{S, T \in L}=\prod_{S \in L} p(\lambda, S) .
$$

Suppose $G$ is $k$-colorable. Then $p(k, \phi)=$ chromatic polynomial of $G$ is $>0$. If, for example, $G$ is critical, in the sense of Dirac, then $p(k, S)>0$ also, for we can color $G-S$ in $k-1$ colors and all of $S$ as the $k$ th, so the matrix or the left is nonsingular. Suppose $G$ is not $k$ colorable. Then $p(k, \phi)=0$ and the matrix is singular. Hence, the chromatic number of $G$ never exceeds the least $k$ for which the matrix is nonsingular, and if $G$ is critical, is equal to it.

\section{REFERENCES}

1. G. F. Clements and $B$. Lindstrom, $A$ sequence of $( \pm 1)$-determinants with large values, Proc. Amer. Math. Soc. 16 (1965), 548-550.

2. B. Lindstrom and $\mathrm{H}$. O. Zetterström, $A$ combinatorial problem on the $k$-adic number system, Proc. Amer. Math. Soc. 18 (1967), 166-170.

3. G. Pólya and G. Szegö, Aufgaben und Lehrsätze aus der Analysis, Berlin, 1964.

4. G. C. Rota, On the foundations of combinatorial theory. I: Theory of Möbius functions, Z. Wahrscheinlichkeitstheorie und verwandte Gebiete 2 (1964), 340-368.

5. G. D. Birkhoff, $A$ determinant formula for the number of ways of coloring a map, Ann. of Math. II Ser. 14 (1913), 42-46.

University of Pennsylvania 